\title{
Formar ciudadanos en centros para menores privados de libertad... Un reto complejo para la educación
}

Adelmo Sabogal Padilla ${ }^{1}$

\section{Resumfen}

Este informe se fundamenta en una investigación realizada sobre el proyecto "La noción de ciudadanía en menores infractores privados de libertad que hacen parte de procesos reeducativos", desarrollada por Adelmo Sabogal Padilla entre los años 2008 y 2009. El problema de investigación abordado está relacionado con la tensión existente frente a la decisión de privar de la libertad a un sujeto que la misma sociedad ha educado. Esto nos puede llevar a preguntarnos no solo por las fall las del sujeto sino por las del sistema. El tema en que confluye esta problemática tiene que ver con las nociones de ciudadania, gue posee la población de menores infractores privados de la libertad. El objetivo propuesto pretendio caracterizar las nociones de ciudadanía de un grupo de jóvenes infractores que hacen parte de procesos reeducativos. También se relacionaron las situaciones a las que atribuyen el origen de su infracción. Los resultados centraron su atención en relación con las siguientes categorías: a) pertenencia nacional, b) reconocimiento como sujetos de derechos y deberes, c) conciencia de pertenencia a una colectividad, d) participación social e integración, e) responsabilidad del buen funcionamiento de las instituciones, f) derechos y deberes correlativos, g) defensa de los propios derechos.

Palabłas clave: Infractor, ciudadanía, reeducación, noción, libertad, pedagogia.

1 Normalista, Licenciado en Pedagogía Reeducativa con énfasis en ética, Magister en Educación en la línea de educación para el conocimiento social y político y estudiante en segundo año de Doctorado en Tecnología Instruccional y Educación a Distancia. E-mail: adelmo.sabogal@unad.edu.co. 


\title{
Educating citizens in juvenile detention centers - A complex educational challenge
}

\begin{abstract}
This research report is based on the project "The notion of citizenship in juvenile offenders who are part of reeducation processes in detention houses" developed by Adelmo Sabogal in 2008 and 2009. The research is related to the tension arisen from the decision to deprive of freedom a subject who was educated by society itself. That can lead us to ask ourselves not only about that individual's failures but al so the system's. The topic at hand here has to do with notion of citizenship that in this particular case refers to the population of juvenile offenders deprived of freedom. The proposed objective sought to characterize the notion of citizenship among a group of young offenders, who are involved in reeducation processes. The situations contributing to the origin of this offense were also listed. The results were focused on the following categories: a) the sense of national belonging, b) their acknowledgement as individuals with rights and duties, c) their awareness of belonging to a collectivity, d) social participation and integration, e) responsibility on the proper functioning of institutions, f) rights and correlative duties, g) protection of human rights.
\end{abstract}

Key words: Offender, citizenship, re-education, notion, detention, pedagogy.

Recibido: 18 Noviembre de 2010

Aceptado: 16 Marzo de 2011

\section{lntroducción}

La pedagogía como ejercicio responsable de la reflexión sobre las teorías que orientan las practicas educativas debe estar presente en donde la necesidad de crecimiento de un grupo social lo requiera. Sin el ejercicio propio de la reflexion pedagogica, nuestras prácticas educativas son solo acciones rutinarias, sin alma, sin debate, sin problemas, sin acuerdos, sin opciones. De esta manera, el pedagogo debe considerarse como un estratega, un individuo atento a escuchar antes de actuar, a investigar para descubrir, a preguntar antes de senalar, a construir en 
Revista de

investigaciones UNAD

Volumen 10. Número 1. Junio 2011

colectivo, a ser prudente y a permitir desde la pedagogía la posibilidad de aclarar, solucionar, planear y proyectar, sin confundir. Por consiguiente, aquel discurso academico polarizado por el poder y por la prepotencia que solo busca convencer a otros, no tiene nada que ver con la pedagogla.

La educación, como el ejercicio consciente de formar a nuevas generaciones, tiene un derrotero claro y es que la sociedad permanezca dentro del marco juridico y político que hemos establecido y que se refleja en el cumplimiento y, respeto de los derechos y deberes para mantenernos en un mundo hasta ahora utópicamente equitativo. Las fallas de estos procesos educativos nos deben llevar a pensar en procesos reeducativos que tienen una doble responsabilidad esencialmente pedagógica, En primer lugar, buscar los procedimientos fallidos en la educación, y en segundo lugar, trazar nuevos derroteros para encaminar al individuo o grupo social que se vio afectado, sin desconocer la responsabilidad sustantiva que tiene la sociedad. Desde esta perspectiva, es posible que el mundo de hoy requiera procesos reeducativos y no educativos.

En la actualidad, y con relación a los centros de reclusión para menores, empiezan a hacerse balances de la aplicabil idad de la nueva Ley de Infancia y A dolescencia con respecto al anterior Codigo del M enor, el cual precisaba en su finalidad "garantizar a los niños, niñas y adolescentes el pleno y armonioso desarrollo dentro de la familia y la comunidad en un ambiente de felicidad, amor y comprension teniendo como referentes la igual dad social y la dignidad humana" (L ey de infancia y adol escencila, A rt. 1). Estos el ementos son constitutivos y marco de referencia de nuestra sociedad actual y su materializacion puede asegurar un cambio de paradigma en la constitucion de procesos sociales mas equitativos para futuras generaciones.

En desarrollo de este proyecto se escucha el testimonio de menores infractores privados de la libertad en un centro de reclusión de la ciludad de Bogotá que estan haciendo parte de procesos reeducativos. El proposito es indagar sobre los marcos nocional es desde donde estos menores conciben la ciudadania y sus distintas dimensiones que - se supone- estan en la base de su constitucion como sujetos politicos. Se parte del hecho de solicitar a los participantes que refieran las condiciones contextuales que los han llevado a cometer dichas infracciones: escenarios, agentes y situaciones, para establecer luego las situaciones sociales que, en la percepcion de los jovenes, han originado la infraccion. A demas se caracteriza la noción de ciudadanía, se analiza si hay relacion o no entre las nociones de ciudadania que explicitan los jovenes con las situaciones que argumentan como origen de su infraccion, y finalmente se establecen en conjunto sus imaginarios colectivos de ciudadania.

Algunos de los motivos que llevaron al investigador a indagar sobre la noción de ciudadania en jovenes infractores se relacionan, en primer lugar, con el potencial politico-participativo de los sujetos alli recluidos; en segundo lugar, con la 
Adelmo Sabogal Padilla

Formar ciudadanos en centros para menores privados de libertad... Un reto complejo para la educación, artículo producto de la investigación

exploración del desarrollo de las dimensiones de ciudadanía en sujetos que han vivido situaciones sociales dificiles y su relacion de coherencia o no respecto al contexto ofrecido por la puesta en practica de politicas sociales de infancia y juventud; y, finalmente, con el interes por la relacion entre infraccion, contexto, minoria de edad y desarrollo de la identidad ciudadana.

También motivó la presente investigación reflexionar en torno al impacto de los cambios de paradigma que se han intentado desde el antiguo Código del M enor (1989), hasta la actual Ley de infancia y adolescencia (Ley 1098 de 2006). En el anterior codigo de infancia los paradigmas estaban orientados por la peligrosidad de los hechos cometidos, en tanto que en el actual los paradigmas establecen tres campos de estructuracion. El primer campo, la protección integral, se entiende desde el reconocimiento del sujeto como, persona de derechos, inmerso en una sociedad que debe garantizar el cumplimiento de los mismos, la prevencion de amenazas y la seguridad de su restablecimiento, para lo cual la protección integral se materializa en politicas, planes, programas y acciones. El segundo campo es la prevalencia de los derechos de los menores, donde se pone de relieve la importancia de que el menor sea respetado en el conjunto de sus derechos y en especial cuando exista algun conflicto o riesgo de vulnerabilidad. Finalmente, el tercer campo, la corresponsabilidad, seentiende como los agentes corresponsables que garantizan los derechos del nino y de la nina, entre los que se mencionan el Estado, la familia y la sociedad. De esta manera parece que se busca generar un fortalecimiento de las politicas de proteccion al menor desde una perspectiva de derechos, no solo en el ambito familiar, sino principalmente en el estatal, dentro de la optica de la responsabilidad social.

\section{Metodología}

La investigación desarrollada se fundamenta en principios de inclusión social y analisis crítico, social y refflexivo, de la realidad. La sociedad necesita repensar sus procesos de socialización, las causas y consecuencias de sus maneras de proceder, la toma de conciencia de sus problemas, su evolucion y la transformacion de microcontextos que se encuentran marginados total o parcialmente del colectivo macrosocial, Este es un ejercicio investigativo inspirado en los propositos mencionados con anterioridad que pretende reflexionar sobre las prácticas sociales reeducativas actuales. El posible impacto dependerá de que las instituciones reeducativas centren su atencion en este tipo de reflexiones que favorecen la provección de la educacion a la ciudadania desde valores de inclusion, solidaridad y equidad.

El tipo de investigación fue cualitativo teniendo en cuenta que se abordan metodologias basadas en principios teoricos tales como la fenomenologia, la hermeneutica y la interaccion social. Se emplearon métodos de recolección 
Revista de

investigaciones UNAD

Volumen 10. Número 1. Junio 2011

de datos que son no cuantitativos, con el propósito de explorar las relaciones sociales y describir la realidad tal como la experimentan en este caso los menores infractores de una escuela de trabajo (Rodríguez, Gil \& Garcla, 1996).

La población encuestada está siendo atendida en la modalidad de privación de libertad, que busca desarrollar un proceso de atencion integral encaminada a generar condiciones para un optimo desarrollo humano desde la finalidad protectora, educativa y restaurativa, previniendo aśl la ocurrencia de nuevas infracciones, de acuerdo con la comprobacion de su responsabilidad penal.

La muestra seleccionada es, en un primer momento, intencionada debido al rango de edad establecido (entre los 14 y 17 años), teniendo en cuenta que las, preguntas tienen componentes cognitivos que garantizan una mejor comprensión durante las entrevistas. En segundo lugar se eligieron al eatoriamente cinco jovenes para hacerles entrevistas en profuncildad, uno por cada ano de edad (14, 15, 16 y 17). Se desarrollo una entrevista focalizada, semiestructurada, que consto de once preguntas abiertas orientadas a averiguar, sobre los componentes conceptuales definidos como dimensiones de ciudadanía. La informacion se registra en voz y se transcribe para efectos de categorizacion y analisis.

Tabla 1. Preguntas de la entrevista y relación con las categorías orientadoras de la investigación.

\begin{tabular}{|c|c|c|c|}
\hline \multicolumn{2}{|r|}{ PREGUNTA PRINCIPAL } & \multirow{2}{*}{$\begin{array}{c}\text { PREGUNTAS DE } \\
\text { APOYO }\end{array}$} & \multirow{2}{*}{\begin{tabular}{|c|} 
ARTICULACIÓN \\
TEÓRICA CON LAS \\
DIMENSIONES DE \\
CIUDADANÍA \\
Contextualización inicial \\
\end{tabular}} \\
\hline 1 & $\begin{array}{l}\text { ¿Cuál es su edad, gustos, } \\
\text { habilidades? }\end{array}$ & & \\
\hline 2 & $\begin{array}{l}\text { ¿Cuáles son los días de visita? } \\
\text { ¿Quién lo visita? ¿Cómo es } \\
\text { su familia? ¿Cómo le iba en la } \\
\text { escuela? ¿Ha tenido buenos } \\
\text { amigos o amigas? ¿Qué le han } \\
\text { aportado? }\end{array}$ & $\begin{array}{l}\text { ¿De su familia con quién se } \\
\text { lleva mejor y con quién tiene } \\
\text { mayor dificultad de relación? } \\
\text { ¿Por qué? }\end{array}$ & $\begin{array}{l}\text { Contextualización de la } \\
\text { infracción }\end{array}$ \\
\hline 3 & $\begin{array}{l}\text { ¿Por qué cometer la } \\
\text { infracción? } \\
\text { ¿Qué razones existían? } \\
\text { ¿Cómo ocurrió? ¿Qué conse- } \\
\text { cuencias le ha traído? }\end{array}$ & $\begin{array}{l}\text { ¿Cuánto tiempo lleva en el } \\
\text { centro; cuánto le falta para } \\
\text { recobrar la libertad? }\end{array}$ & $\begin{array}{l}\text { Contextualización de la } \\
\text { infracción }\end{array}$ \\
\hline 4 & $\begin{array}{l}\text { ¿Usted se considera colom- } \\
\text { biano } \\
\text { (a)? ¿Por qué? }\end{array}$ & $\begin{array}{l}\text { ¿Qué le gusta o le disgusta } \\
\text { de su país? }\end{array}$ & Pertenencia nacional \\
\hline
\end{tabular}




\section{Adelmo Sabogal Padilla}

Formar ciudadanos en centros para menores privados de libertad... Un reto complejo para la educación, artículo producto de la investigación

\begin{tabular}{|c|c|c|c|}
\hline \multicolumn{2}{|r|}{ PREGUNTA PRINCIPAL } & $\begin{array}{c}\text { PREGUNTAS DE } \\
\text { APOYO }\end{array}$ & $\begin{array}{c}\text { ARTICULACIÓN } \\
\text { TEÓRICA CON LAS } \\
\text { DIMENSIONES DE } \\
\text { CIUDADANÍA }\end{array}$ \\
\hline 5 & $\begin{array}{l}\text { ¿Cuál es su concepto de dere- } \\
\text { cho y de deber? ¿Cuáles son } \\
\text { sus derechos? ¿Cuáles son } \\
\text { sus deberes en el centro de } \\
\text { reclusión y en la ciudad? }\end{array}$ & $\begin{array}{l}\text { ¿Cuál es su concepto de la } \\
\text { palabra "ley" y de las institu- } \\
\text { ciones que la representan? }\end{array}$ & $\begin{array}{l}\text { Reconocimiento como suje- } \\
\text { tos de derechos y deberes }\end{array}$ \\
\hline 6 & $\begin{array}{l}\text { ¿Cree que el acto que cometió } \\
\text { afectó a otras personas? ¿Lo } \\
\text { haría nuevamente? ¿Por qué? }\end{array}$ & $\begin{array}{l}\text { ¿En el momento de la } \\
\text { infracción era consciente } \\
\text { del daño que hacía? ¿Ahora } \\
\text { es consciente del daño que } \\
\text { pudo haber causado? }\end{array}$ & $\begin{array}{l}\text { Conciencia de pertenencia a } \\
\text { una colectividad }\end{array}$ \\
\hline 7 & $\begin{array}{l}\text { ¿De dónde es usted, dónde } \\
\text { nació? ¿Cómo es por allá? } \\
\text { ¿Cómo es la gente? }\end{array}$ & $\begin{array}{l}\text { ¿Cómo se encuentran orga- } \\
\text { nizados en el centro? ¿Qué } \\
\text { grupos existen en el centro y } \\
\text { qué funciones tienen? }\end{array}$ & $\begin{array}{l}\text { Participación social e inte- } \\
\text { gración }\end{array}$ \\
\hline 8 & $\begin{array}{l}\text { ¿Cree que en la ciudad en } \\
\text { que vive existen los mismos } \\
\text { grupos u organizaciones que } \\
\text { funcionan en el centro? ¿Las } \\
\text { actividades de las que son } \\
\text { responsables en estos grupos } \\
\text { son desarrolladas de manera } \\
\text { consciente u obligada? }\end{array}$ & $\begin{array}{l}\text { ¿Existe cuidado entre los } \\
\text { integrantes del centro de } \\
\text { menores? } \\
\text { ¿Cómo eligen a sus líderes? } \\
\text { ¿qué respeto tienen hacia } \\
\text { ellos? }\end{array}$ & $\begin{array}{l}\text { Responsabilidad del } \\
\text { buen funcionamiento de las } \\
\text { instituciones }\end{array}$ \\
\hline 9 & \multicolumn{2}{|c|}{ ¿Considera que todo derecho tiene un deber? } & $\begin{array}{l}\text { Derechos y deberes corre- } \\
\text { lativos }\end{array}$ \\
\hline 10 & $\begin{array}{l}\text { ¿Ha participado en alguna } \\
\text { lucha o manifestación? ¿Cuál? } \\
\text { ¿Por qué? }\end{array}$ & $\begin{array}{l}\text { ¿Qué pasa en el centro } \\
\text { cuando no cumplen con sus } \\
\text { deberes? } \\
\text { ¿Afecta esto sus derechos? }\end{array}$ & $\begin{array}{l}\text { Defensa de los propios } \\
\text { derechos }\end{array}$ \\
\hline 11 & $\begin{array}{l}\text { ¿Cuál es su concepto de } \\
\text { ciudadanía? }\end{array}$ & $\begin{array}{l}\text { ¿Cuál es su concepto de } \\
\text { ciudad? }\end{array}$ & Noción de ciudadanía \\
\hline
\end{tabular}

\section{Resultados}

A continuación se presentan los resultados y discusiones de las entrevistas que permiten asociar, comparar y establecer puntos de encuentro y desencuentro entre las respuestas dadas a las diferentes categorías. En resultados y discusión uno se presenta la sintesis de los datos recol ectados en los portafol ios de cada entrevistado y el analisis correspondiente a la lectura integral de la informacion. Resultados y discusión dos surge de las respuestas de los entrevistados y facil ita comprender el contexto de la infraccion cometida, desde una mirada social, familiar, escolar, de pares y se relaciona con el lugar de privacion de libertad. De esta misma manera 
Revista de

investigaciones UNAD

Volumen 10. Número 1. Junio 2011

se analizan las respuestas con relación al marco teórico planteado. Finalmente, en resultados y discusión tres se retoman las categorías de ciudadanía referidas en el marco teórico y se detallan los puntos de encuentro y desencuentro de los entrevistados con relacion a los conceptos planteados. A continuacion se presenta de manera separada cada uno de los tres apartes de Resultados y discusión:

1. Sintesis de los datos recolectados. Los entrevistados ya han pasado por la etapa de la pubertad (12 a 13 años), aunque se parte de que la condicion de juventud no es uniformey varia de acuerdo con el grupo social que se considere. Es importante tener presentes actitudes propias de la edad y que influyen directamente en las categorías estudiadas relativivas a la formacion de ciudadania como busqueda de identidad, consolidacion, de amistades afectivas, experimentacion grupal y autoafirmacion. Ellas son imprescindibles para la madurez social, a menudo rodeada de conflictos, resistencia y/o interferencia con su independencia.

Se evidencia de inmediato en los datos de los entrevistados el predominio de familias reconștituidas (dos de los cinco casos) y familias monoparentales con jefatura femenina (dos casos). Al gunos que tienen padrastros no conocen a sus verdaderos padres. En las famil ias que son reconstituildas, el numero de hermanos y hermanas es amplio. En el proceso de reconstitucion familiar la madre es la que se mantiene en el hogar. Solo uno de los encuestados aun mantiene su familia nuclear y uno tiene como familia a su hermana.

Cuatro de los menores entrevistados llegan al centro de privación de libertad sin haber sido reincidentes;' en el unico caso que reincide, es juzgado ahora por una infracción más severa. L as infracciones indican una tendencia al acceso de dinero de manera ilicita, bien sea directa o indirectamente. L a directa se refiere a intereses individual es y la indirecta a fines colectivos, por ser otros intermediarios quienes se benefician del acto.

Los menores infractores tienen orígenes de nacimiento en municipios o provincias circundantes a la ciudad de Bogota. Tres de los encuestados han migrado de su ciudad de origen, dos de ellos a la Capital y uno al municipio de Soacha, Cundinamarca. Dos se mantienen en sus provincias o municipios de origen.

El tiempo de privación de libertad lo estableceel juez de menores pero está sujeto al comportamiento y avance en el proceso reeducativo que demuestra el infractor, que se puede extender si los reeducadores solicitan la ampliación de tiempo atendiendo a los conceptos de trabajadores sociales, psicólogos y, en general, del equipo interdisciplinario.

Los asuntos anteriores están relacionados con las reformas estructurales entre la ley anterior y la nueva ley de infancia y adolescencia. Al pasar del Código del Menor a la Ley de Infancia y Adolescencia, se pasa de una normatividad que habla de situaciones irregulares que enfrentan a l gunos niños, niñas y adol escentes, 
Adelmo Sabogal Padilla

Formar ciudadanos en centros para menores privados de libertad... Un reto complejo para la educación, artículo producto de la investigación

a una de protección integral. Esto supone un vuelco institucional en tanto el Estado debe garantizar los derechos de todos ellos. Consecuentemente, se debe redefinir el papel del Sistema Nacional de Bienestar Familiar desde el ámbito nacional hasta los ambitos departamentales, distritales y municipales. Se puede decir que todas las entidades publicas deberan ahora garantizar, de acuerdo con sus competencias y obligaciones, los derechos de los ninos, las ninas y adolescentes. No se trata sin embargo, de grandes reformas administrativas sino de ajustes en las instituciones que deben vel ar por el cumplimiento de esos derechos. La Escuela de trabajo "El Redentor" ha adoptado, particul armente, programas de prevencion y proteccion integral a los menores. Sin embargo, quienes fueron juzgados bájo la ley antigua no pueden gozar de los beneficios y programas de la nueva ley y, por tanto, deben terminar su proceso tal como fueron juzgados.

En relación con los datos de escolaridad de los entrevistados es también evidentel a falta de preparacion escol ar, lo que incide en las escasas habilidades propias como la escritura, los trazos en dibujos y la expresión verbal. sorprende al mismo tiempo el amplio conocimiento experiencial en los campos correspondientes a la infracción de normas o a la comisión de ilícitos.

2. Respuestas de los entrevistados. L os lazos afectivos con las madres son los que mas se mantienen en estos momentos, y ello se transforma en un fuerte motivo para desear recobrar la libertad. Las dificultades del menor son general mente con los padrastros, ya que no aceptan con facilidad decisiones trascendentales para una pareja como la separacion. Este es un hecho tan significativo que provoca efectós importantes como retiro del ámbito escolar o falta de control en las responsabilidades que los menores tenían en su hogar.

Inicialmente los menores no cuentan abiertamente las infracciones cometidas. Se destaca descuido y apatía hacia las actividades escolares, principal mente en el cumplimiento de horarios en el colegio o tareas asignadas por los profesores. Cuando el menor ha vivido primero con uno de los padres y despues con el otro, las costumbres que uno de ellos tenía, sobre todo si era permi sivo, quedan más registradas en las acciones del menor. Por esta razon explora espacios como sálas de billar u otros lugares donde pueda pasar el tiempo.

Los esfuerzos que el menor intente hacer por continuar actividades como sus estudios carecen de sentido sin el apoyo familiar. En los casos en que los padres no han ejercido control preventivo desde un principio y lo intentan hacer cuando notan cambios fuertes en sus hijos, ellos ya no lo aceptan y tienden a irse de su casa. Los hermanos fortalecen sus lazos afectivos debido a que viven y comparten la misma sittuacion familiar. Cuando son medio-hermanos también es evidente esta relación, debido a que se mantiene el abuso de fuerza 0 autoridad de al guno de los padres. 
Revista de

investigaciones UNAD

Volumen 10. Número 1. Junio 2011

Lasituación familiar, aun con sus particularidades, afectadi rectamente el estado de animo del menor y acrecienta las posibilidades y razones para volver a la libertad. Es evidente que en los procesos de resocializacion la resignificación de la estructura familiar recobra fuerza en estos momentos dificiles, debido a que existe reconocimiento bilateral entre padres e hijos adolescentes de la importancia y necesidad de la existencia de las dos partes en la dinamica tamillar, En conclusion, en la actual idad tanto hijos como padres representan figuras importantes e igualmente necesarias en el hogar.

El énfasis de las habilidades se asocia con actividades que se realizan en los contextos inmediatos que, en este caso, están relacionados con las acciones reeducativas de la Escuela de trabajo "El Redentor", las actividades musical es y aquellas centradas en talleres de posibilidad laboral, que son los mas nombrados.

Las actividades preferidas para expresar sentimientos de identidad 0 inconformidad son las relacionadas con la musica, debido a que no existen senalamientos que impidan esta libre expresion. A demas es una forma de posicionarse en un grupo, llamar la atencion y ser reconocido publicamente.

La asistencia a las escuelas académicas formales se transformó en mecanismo para evadir las responsabilidades y esconderse de los profesores o los padres, generalmente cambiando de barrios. LOS padres optaron por estrategias de estudio en horario nocturno, pero la dificultad no radica en el horario sino en la motivación y en los hábitos de estudio. En estas circunstancias el joven prefiere entrar a trabajar, pues dispone de dinero en una sociedad de consumo que le ofrece al adolescente oportunidades para integrarse a otros grupos de jovenes 0 adultos.

Es interesante destacar que el concepto de grupo está asociado a más de ocho integrantes, y que se expresa mayor confianza en los colectivos, de dos o tres personas. En el centro, el valor de la confianza es uno de los más importantes debido a que a los menores que lo demuestran se les otorgan responsabilidades de liderazgo y cuidado grupal. Se organizan por colectivos, de los mas resistentes a los más dociles, que se han apropiado con mayor facilidad del proceso reeducativo. Algunos menores que han recobrado su libertad pero otra vez son juzgados terminan en un alto porcentaje en la cárcel de La Picota, donde ya son reconocidos por sus múltiples infracciones.

Tanto la infracción como la reincidencia hacen pensar en la necesidad de proponer, al gun tipo de intervencion sopre las concepciones y practicas de ciudadanía de los jovenes, dada su relacion directa con el reconocimiento de si mismos como sujetos de derechos y deberes.

La proyección de los menores al salir del centro apunta a recuperar las posibilidades laborales y a que sean aceptados sin complicaciones. El primer escenario para lograrlo es la actividad laboral de los padres. 
Adelmo Sabogal Padilla

Formar ciudadanos en centros para menores privados de libertad... Un reto complejo para la educación, artículo producto de la investigación

El grupo de infractores, independientemente del nivel de gravedad del delito por el que son juzgados, ya es identificado y estigmatizado en los barrios por, los familiares, la policia, los jueces, etc., lo que constituye un imaginario dificil de cambiar.

Los menores comprenden que para borrar esta marca deben autocontrolarse en sus correspondientes contextos pues alla, a recobrar la libertad, encontraran de nuevo grupos que los pueden llevar otra vez a consumir sustancias psicoactivas 0 a cometer infracciones. Algunos de ellos consideran que la ley es injusta pues pareciera un asunto del azar, ya que al gunos caen pero otros continuan afuera delinquiendo. En conclusion, los menores privados de la libertad son apenas una minoria en comparacion con los que estan afuera.

El apoyo del colectivo de amigos en este tipo de experiencias se puede perder pero el de la familia es posible que se resignifique. Las experiencias vividas y que causaron la perdida de libertad ensenan, al menos teoricamente, que conseguir dinero fácil no es el camino correcto, que trabajar legal mente seria lo adecuado; aun asi, esta eleccion no es una garantia en una tensa situacion donde confluyen factores como las drogas, el alcohol, la desarticulacion familiar, la falta de apoyo social y el desempleo, entre otros.

Los menores consideran que la mejor forma para hacer entender y comprender preventivamente que no es bueno hacerle dano a otros o a sí mismos es contar a historia de vida y util lizarla como recurso pedagogico en la educacion formal. Quienes cometen infracciones son reconocidos en sus barrios como ladrones 0 simplemente como jovenes que estan en "mal os pasos". Para ellos, pertenecer a estos col ectivos proporciona ciertos niveles de seguridad debido a que se puede transitar por los barrios con articul os como oro, chaquetas de cuero o altas cantidades de dinero. Existe una compul sión de la sociedad por el consumo, y siendo un sistema globalizado que prioriza el dinero como medio de desarrollo, la presion es mas fuerte para este tipo de poblacion.

La policía de menores es la encargada de capturar y acompañar a estos menores hasta el juez y es quien presenta las pruebas del caso y las armas o el material incautado. De esta manera se asume que el personal humano que compone la policia fundamenta su actuacion en valores como honestidad, transparencia y buen trato en el cumplimiento del procedimiento con menores, garantizando sus derechos y deberes. En contravía de lo mencionado anteriormente y anal izando los testimonios, todos los menores entrevistados individual mente coinciden en que algunos policias no son buenos representantes de justicia debido a que en ocasiones incluyen, por ejemplo, pertenencias (armas cortopunzantes, sustancias psicoactivas, entre otras) que eran de otra persona en el momento de la captura y las presentan ante el juez como prueba. Se puede afirmar que se busca, prevenir y atender estos hechos desde el juzgamiento delincuencial declarandolos sujetos peligrosos para la sociedad pero no 
Revista de investigaciones UNAD

Volumen 10. Número 1. Junio 2011

se fomentan programas que busquen el desarrollo integral de los grupos de adolescentes en su ciudad. A pesar de que el menor tenga una versión diferente de los hechos, se asume que la policia dice la verdad y que el menor no. Los supuestos infractores se quejan de que los procesos de investigacion no son mas constantes y profundos para verificar los testimonios presentados por las partes e incluso af irman que el denunciante dice cosas que no corresponden a la realidad para que la infraccion resulte de mayor peligrosidad. Tambien hay evidencia de casos de racismo o segregacion, debido a que por el color de piel o por la forma como se visten reciben maltratos verbales o humillaciones.

En lo que respecta a las relaçiones al interior de la institución reeducativa, algunos menores dicen asumjr una actitud más agresiva a su ingreso para evitar que el grupo que ya esta en la institución desde tiempo atras, no intente "montârsela". Estos comportamientos pueden desencadenar hechos violentos 0 agresivos de al gunos jovenes que judicialmente se pueden afectar, ya que al asumir actitudes de intol lerancia, si se produce al guna gresca general se altera el tiempo de permanencia en la institucion. De esta manera es evidente que el trato excluyente y estigmatizante se da entre los mismos jovenes, lo que sugiere al centro de reclusion y al Estado en general la necesidad de centrar los proyectos social es e institucionales en la implementacion de practicas sociales flexibles, abiertas e incluyentes.

Es definitivo que el mayor derecho, que se valora por encima de cualquier tipo de comodidad, es la libertad. Al restringirse, uno de los menores expresa que los, sentimi entos que emergen son de deuda con la sociedad, y que con su reclusion, literalmente "se esta pagando al go".

Existe respeto por los reeducadores que son más estrictos y que aplican estrategias acertadas para que las normas acordadas se cumplan; los menores los asocian con sus padres, pero la figura de autoridad del reeducador es mas significativa debido a que desde el inicio del proceso en la Escuela de trabajo "El Redentor", definieron limites y reglas que cumplir.

En el centro constantemente se habla del ambiente que se vive en una cárcel de adul tos. Familiares o amigos dan su testimonio y desde esta perspectiva los menores valoran estar en la Escuela de trabajo "El Redentor", viviendo en condiciones humanas, ya que perciben la carcel de adul tos como un lugar en donde todas las relaciones dependen del dinero.

Las actividades de integración pueden ser más significativas en tanto se realicen mas continuamente, enfocadas como actividades academicas de construccion colectiva, bien sea desde el trabajo en talleres 0 en donde existan retos academicos que cumplir y la metodología de clase se fundamente en la adquisicion y evaluacion de competencias. 
Adelmo Sabogal Padilla

Formar ciudadanos en centros para menores privados de libertad... Un reto complejo para la educación, artículo producto de la investigación

3. Categorías de ciudadanía. Se denotan dos niveles de pertenencia nacional. El primero, relacionado con la territorialidad, hace referencia a nacer en un lugar determinado. El segundo nivel representa una conexion importante con ciudadania social, pues al expresar querer cambiar de pais por una razon tal como la tal ta de oportunidades labora es para menores, se reconoce la ciudadania social como el contexto donde el Estado debe ofrecer bienestar colectivo y sentido comunitario para toda la sociedad sin ninguna clase de exclusiones. Por lo anterior es importante mencionar que el Estado, como ente gubernamental, debe garantizar el bienestar de los ciudadanos e interesarse por mantener un sistema solidario donde los menores manifiesten expresamente su deseo de concebir la sociedad como una totalidad donde todas las personas tengan el mismo derecho a la calidad de vida, en especial al acceso educativo, evitando una clasificación social acorde con el lugar donde se estudie.

Se puede concluir que el proceso de formación respecto a la dimensión de reconocimiento como sujeto de derechos y deberes, no es relevante segun las respuestas de los encuestados. Las experiencias que los han llevado a reconocerse como sujetos politicos estan enmarcadas por la sqnción judicial por el incumplimiento de sus propios deberes. La resignificacion de comprender que se es sujeto de derechos y deberes se fundamenta, ironicamente, en que la sancion vul nera uno de los derechos civiles mas importantes de la humanidad, el derecho a la libertad.

Existe una conciencia desarrollada para ponerse en el lugar del otro y en este caso de la ciudad, cuando se afirma que los derechos y la facilidad de acceso a estos, que claramente se ofrecen desde el centro de menores, deberian proyectarse a la ciudad. De acuerdo con el desarrollo moral de K hol berg, se podría mencionar que esta es una posible evidencia de mutual idad, etapa tres (3) del nivel dos (2), moral convencional, en donde la perspectiva consiste en ponerse en el lugar del otro: es el punto de vista del individuo en relacion con otros individuos, en el que se destacan los sentimientos, acuerdos y expectativas compartidas, pero no se llega todavia a una generalizacion del sistema.

En relación con los deberes, se denota una concepción de responsabilidad unicamente con el circul o de convivenciainmediato. Es claro queel incumplimi iento con los deberes que ha afectado a otros ciudadanos es la razon por la cual se ha perdido el derecho a la libertad, pero existe de manera directa una exigencia a os representantes de la ley, en este caso a la policía nacional, para que como inherentes portadores de la verdad y la justicia, actuen de manera coherente con su natural eza. En todos los entrevistados existen experiencias que marcan la falta de credibilidad en los representantes de justicia.

El derecho a la libertad es el que definitivamente representa la mayor importancia en rel acion con otros derechos, pero la necesidad de orientacion en los procesos de socialización en la escuela, la familia y la sociedad, hace que cuando el contexto 
Revista de investigaciones UNAD

Volumen 10. Número 1. Junio 2011

no garantiza con calidad los mismos derechos y deberes, obliga a que desde el quebrantamiento de la ley se comprenda la importancia y la funcionalidad de los derechos y deberes en relación con nuestra natural eza humana.

El estado de conciencia en el momento de cometer la infracción es diferente del que se tiene cuando se esta cumpliendo la condena. El menor que comete el ilicito simplemente actua sin ningun tipo de contemplación no piensa en el estado económico, género, edad ni ningún otro rasgo del afectado. El fin individual es el único obiétivo que cumple por presion de terceros, por efectos del consumo 0 por voluntad propia. Despues de ser capturado, escuchando testimonios de las victimas, la conciencia colectiva aparecey los sentimientos de igualdad y respeto por los derechos colectivos adquieren importancia. De esta manera se puede concluir que es necesario educar en la conciencia colectiva con responsabilidad y corresponsabilidad.

Dos elementos importantes surgen en el análisis de la categoría "Participación social e integración :

1. La asociación y agrupación de estos menores, como adolescentes que son, ocurre de manera abierta y descomplicada. En estas relaciones juveniles, la formación que se ha dado desde el hogar se somete a prueba debido a que las formas de asociación pueden tener diferentes fines que ponen en juego el proceso de aprendizaje ciudadano que se ha estructurado desde la Familia, el Estado, la escuela y la sociedad. En los jovenes entrevistados se reconoce una gran debilidad en estos aprendizajes familiares y sociales, que necesariamente desencadenaron decisiones que provocaron el rompimiento de las normas estatales y causaron la infraccion por la cual hoy son juzgados.

2. Los grupos a los que han pertenecido estos menores los han llevado a cometer ilicitos. Este hecho era el fin de los respectivos colectivos, es decir, la organizacion al cometer ilicitos cumple su objetivo. En la dinamica interna existe una jerarquía clara, incuestionable, normativa y rentable, frente a la cual el joven infractor debe cumplir compromisos como estos: a) horas especificas de reunion, b) actividades asociadas a diverșion y a ganancias economicas rentables segun una estructura organizacional rigurosa, c) consumo de sustancias psicoactivas, d) asumir riesgos que se conocen desde un principio, tales como perdida de seres queridos, carcel, abuso del consumo de sustancias psicoactivas o del al cohol, o la perdida, de la vida misma, e) organizacion economica del colectivo por medio de líderes que pagan mensualmente, dependiendo de lo que el gran grupo ha logrado en el mes, f) ser sancionado, por ejemplo con la exciusion del grupo și es capturado por la policia, g) hacer estudios previos para cometer los ilicitos.

Es importante destacar que la participación social e integración del joven a la organización de carácter ilegal dedicada a cometer ilícitos se fundamenta en 
Adelmo Sabogal Padilla

Formar ciudadanos en centros para menores privados de libertad... Un reto complejo para la educación, artículo producto de la investigación

las relaçiones de poder que están centralizadas en la adquisición de dinero, el reconocimiento de otros con mayor jerarquia, la independencia en la toma de decisiones cuando las cosas salen mal y el consumo sin restriccion. Estos aprendizajes sociales, algunos basados en el adultocentrismo productivo, que se han trasmitido por generaciones, son los causantes de las infracciones cometidas por los menores. Aun mas, son desatadas, por la falta de programas y proyectos de intervencion en la escuela y en los barrios que potencialicen la participacion y organizacion de los jovenes en sus contextos ciudadanos. Otra seria la situacion, pues estos mecanismos permitirian asi el surgimiento de diversas estructuras microsociales de gran impacto ciudadano.

La escasez de estos proyectos de organización juvenil está fortaleciendo la cultura de la ilegalidad, respecto de lo cual' Saúl Hernandez Bolivar dice:

"Nuestra sociedad ha perdido sus valores en pos de un enriquecimiento rápido e illimitado o de la mera supervivencia. La cultura popular está tan permeada por ese fenómeno que se refleja una manera de pensar opuesta a la de nuestros abuelos, el honor no vale nada, se destaca más la picardia, la viveza, la malicia. Se ap laude al avispado y el virtuoso es tenido por idiota; se da por hecho que no hay que respetar lo ajeno. Hasta tal punto han llegado las cosas que cuando un nino muy pobre de M edellin devolvio una gruesa suma de dinero que se encontro en el suelo, el asesinado gobernador de Antioguia, Guillermo Gaviria, lo premio con una casa pero no por su acto de honestidad - un acto normal que no deberia merecer un premio - sino porque al gobernador le pareció muy doloroso saber que el niño se habia vuelto centro de burlas en su barrio" (www.elmundo.com).

Los menores reconocen la relación entre derecho y deber, pero la entienden en el sentido de que los deberes son una categoria dentro de los derechos, y que la sancion hace parte de esa relacion entre derecho y deber.

Sería entonces pertinente trabajar para crear conciencia de la necesidad de cumplir derechos y deberes dentro de un marco que facil ite la sana convivencia, la participacion ciudadana y el respeto por los valores. Lo que se debe focalizar en el trabajo con los jovenes es la reivindicacion de la participacion individual y colectiva, institucionalizada o autónoma, en busca del bien comun. Esto implica acogerse a un grupo de modelos normativos acordados previamente por otros, colectivos, con la posibilidad de socializacion y debate para su apropiación o reestructuracion, utilizando mecanismos legales y democraticos de participacion o protesta.

La ciudadanía se concibe como el conjunto de deberes y derechos, con niveles de participacion en las instituciones del Estado, reconociendo en ell las una posibilidad de proyeccion personal y profesional. A su vez existe la idea ético-social que permite pensar en el bienestar col ectivo relacionado con la integridad de la persona 
Revista de

investigaciones UNAD

Volumen 10. Número 1. Junio 2011

y el cuidado de los espacios públicos. La noción se expresa desde ideas simples ejemplificadas, debido a que no ha existido un acercamiento educativo a las categorias que componen el ambito de la formación ciudadana'; el conocimiento ha sido experiencial desde el campo de la infraccion, lo cual permite definirlo global mente aun sin haber recorrido didacticamente sus ejes temaaticos.

El contexto de la ciudadanía se concibe para ciudades grandes e importantes donde existsten posibilidades de trabajar, lo que general mente es una de las razones de migracion de las provincias o zonas rurales. Desde esta perspectiva, el termino ciudadania se asocia al contexto de la ciudad (territorialidad) y no a un conjunto de competencias incluyentes que tenemos también en otros espacios sociales, afectivos y cognitivos.

Lainstitución social que toman como referente los menores para dar sus respuestas es a la que pertenecen en su contexto inmediato, que corresponde al centro de reclusión. Las responsabilidades adquiridas para el cuidado, mantenimiento y participacion se cumplen en la mayoría de los casos por la presion de la sancion y no por el buen funcionamiento de la dinámica cotidiana. Esta dimensión se debe considerar en el desarrollo de una conciencia mas colaborativa.

De igual manera el imaginario sobre los mecanismos de participación vistos en el contexto social tales como el voto, las movilizaciones sindicales 0 las diversas manifestaciones, corresponde a unas dinamicas que carecen de escucha estatal 0 al incumplimiento de promesas politicas ofrecidas en las campañas.

En el centro, los menores se encuentran organizados en grupos de acuerdo con el avance de su proceso reeducativo, y de esta manera se clasifican para compartir las actividades rutinarias y los alojamientos. Cada colectivo tiene un nombre y los grupos mismos se ayudan en su cuidado, es decir existe comportamiento ético-social, segun el cual los jovenes buscan de alguna manera el bienestar global, basándose en preceptos tan el emental es como la justicia, la libertad, la verdad y la equidad.

\section{Conclusiones}

Las situaciones que llevan a los menores a cometer los ilícitos están básicamente relacionadas con tres elementos: la familia, el Estado y la escuela. En primer lugar, la familia no establece los límites necesarios para que el adolescente tenga un referente de autoridad. Esta no se puede entender como el mandato agresivo de los adultoss', es la figura que en coherencia con su actuación ha establecido acuerdos básicos de comportamiento para el beneficio individual y colectivo de la familia, incluyendo posibles sanciones apl icadas oportunamente, que obedecen mas a estrategias creativas, que permiten refl exionar y reconstruir un acuerdo. En 
Adelmo Sabogal Padilla

Formar ciudadanos en centros para menores privados de libertad... Un reto complejo para la educación, artículo producto de la investigación

los casos en que la familia pierde su unidad, es necesario fortalecer el diálogo y la comunicación oportuna con los jovenes, analizando las razones por las que un nucleo familiar consanguíneo puede en determinado momento romper sus lazos, y los beneficios de una nueva dinamica familiar. Se sugiere aca un acompanamiento al adolescente sin restringir sus espacios propios de expresion.

En segundo lugar, el Estado tiene la responsabilidad de desarrollar programas en dos perspectivas encaminadas a garantizar, la participacion del menor y que son: espacios para la creación de movimientos juveniles con fines de participación politica seria con voz y voto, y la reflexion sobre las politicas de trabajo para menores en un contexto que debe considerar a algunos de estos como cabezas de hogar.

La Escuela es responsable de repensar las estrategias, didácticas y pedagógicas que emplea, y la potenciali idad de áreas sociohumanísticas como la formacion ci udadana, para evaluar si real mente los tiempos dedicados a las clases diarias permiten formar integralmente el estudiante y centrar el aprendizaje desde el mundo contextual real.

La noción de ciudadanía de los jóvenes se ha estructurado desde los referentes fundados en el rompimiento de la ley. Esto les permite dar una mirada integral a las dimensiones planteadas a pesar de no tener un programa básico de formacion ciudadana brindado por la escuela, el Estado o la familia. La perspectiva de los menores privados de la libertad y sus desafortunadas experiencias son el punto de partida para comprender y trazar estrategias pol iticas que real mente contemplen todos los contextos sociales y atenderlas realmente. Un programa de formacion ciudadana nacional no se puede estructurar sin la participacion de estos colectivos de jóvenes y a credibilidad en ellos. El concepto de los intelectuales puede carecer de importancia 0 aplicabilidad si no tiene unos referentes reales que visibilicen las diferentes miradas de la comunidad infantil y adolescente.

Existe relación entre las nociones de ciudadanía que explicitan lo jóvenes y las situaciones que argumentan como el origen de su infraccion, pues son conscientes de las dimensiones de pertenencia nacional, reconocimiento como sujetos de derechos y deberes, sentido de pertenencia a una colectividad, participación social e integración, responsabilidad del buen funcionamiento de las instituciones, derechos y deberes correlativos y defensa de los propios derechos. El menor, intenta acceder a sus derechos y deberes', la dificul tad radica en los mecanismos por medio de los cuales lo logra, la orientacion, las oportunidades y las garantias que ofrece el contexto social.

L os imaginarios colectivos sobre las dimensiones de ciudadanía, concretamente se pueden expresar de la siguiente manera: 
Revista de

investigaciones UNAD

Volumen 10. Número 1. Junio 2011

- Pertenencia nacional. Asociado a territorialidad y estado de bienestar.

- Reconocimiento como sujetos de derechos y deberes. En mayor medida se reconocen los derechos pero los deberes son mas dificil es de establecer.

- Sentido de pertenencia a una colectividad. En términos de un menor privado de la libertad, hacer daño a otro ciudadano es posible siempre y cuando el fin personal lo justifique, pero si el agredido es el infractor, surge de manera inmediata la conciencia de respeto por la propia integridad. Esta dimension se restablece al escuchar a los afectados.

- Participación social e integración. Los espacios de participación ciudadana creados por el Estado son escasos. Incluso aś, la vincul ación a colectivos de jovenes se da independientemente de sus fines y dentro de ellos se desarrollan procesos de socializacion.

- Responsabilidad del buen funcionamiento de las instituciones. La funcionalidad propia de las instituciones se piensa a corto plazo; existe una responsabilidad fundamentada en los mecanismos de sancion.

- Derechos y deberes correlativos. Sin ninguna duda se piensa en la coexistencia de los dos y en la responsabilidad del cumplimiento de los deberes, aunque es dificil establecer la adquisicion de derechos.

- Defensa de los propios derechos. No existe una tendencia a participar en movimientos juveniles. La organización en colectivos se piensa desde la motivación de agentes externos como el Estado, la escuela y la familia entre otros, pero no se asume como una oportunidad o un derecho individual.

Esnecesario continuarlainvestigación educativasobrelos procesos de reeducación, enseñanza y aprendizaje, desarrollados en los contextos cerrados y semicerrados con poblacion que ha infringido la ley. Los campos de investigacion pueden estar relacionados con los sistemas de educacion interna, el apoyo del contexto ciudadano en la reeducacion de esta poblacion, los programas de,prevencion, las organizaciones juveniles internas y su impacto social, sus ideologias pol iticas, los procesos de socializacion, la responsabilidad estatal, los niveles de reeducación, os procesos académicos y pedagógicos que estructuran los tall leres desarrol lados, las estrategias docentes, directivas y polititcas, la integracion de los programas desarrollados en estos centros de reclusion con los programas formales, la proyeccion educativa y laboral de la poblacion, los estandares de formación ciudadana propuestos por el MEN en este contexto, a prospectiva educativa de estas instituciones y los modelos pedagogicos utilizados, entre otros.

Las universidades, como gestoras de ideas y campos de investigación, pueden estructurar para sus estudiantes de posgrado cursos afines a las experiencias 
pedagógicas desarrolladas en centros de reclusión, potenciando el grupo de profesionales interesado en la amplia gama de investigaciones posibles en este contexto y que aportan al desarrollo social y cultural de nuestro pals desde la mirada de una educacion incluyente, integral y social.

En el contexto de pregrado, las universidades pueden generar interés y expandir el panorama del campo de accion del futuro licenciado, planteando dinámicas educativas que se puedan desarrollar con esta poblacion y posibilitando la reflexión de teorias de aprendizaje y la aplicacion de modelos educativos desde una mirada integral ciudadana. Presentar una vision de estos contextos puede permitir una mirada educativa en su coniunto y comprender los imaginarios Individuales de muchos estudiantes que atraviesan por situaciones rel acionadas y que pretenden que el educador sea un orientador y posibilitador de caminos que ayuden a mejorar su calidad de vida.

La educación es una sola y los avances que surjan desde el campo tecnológico, politico y social, deben conocerse en los procesos de preparacion de nuestros icenciados y posgraduados. La reflexion educativa y los curriculos deben estar a a vanguardia de estos avances. Existe un riesgo inminente que puede distanciar a los licenciados de los campos de acción que el contexto mundial está generando si no los planeamos, diseñamos, y discutimos desde el escenario academico. En concreto, es en el campo tecnologico donde se pueden hacer preguntas para que las mesas de trabajo profesional reflexionen dentro de su propio contexto, por ejemplo sobre el concepto de plataforma tecnologica, el e-learning, los modelos de aprendizaje que surgen desde los avances tecnologicos, la vinculacion de los avances de la tecnologla a dinámicas pedagógicas, etc.

Es cierto que los programas de especialización pueden permitir una mirada mundial pero la necesidad creativa y didáctica de los procesos académicos desde un punto de vista integral debe estar por lo menos contextual mente presente en los profesionales de la educacion y, mas aun, posibilitar la vinculacion de estas estrategias en beneficio de la real idad educativa que se vive en las aulas de clase, como uno de los escenarios posibles del licenciado.

\section{Referencias bibliográficas}

Almond, G. y Verba, S. (1992). La cultura política. En Diez textos básicos de Ciencia Política (pp.171-201). Barcelona: Ariel.

Arendt, A. (1984). Los derechos públicos y los intereses privados. Ciudad de México: Fondo de Cultura Económica.

Bozhovich, L. I. (1976). La personalidad y su formación en la edad infantil. La Habana: Editorial Pueblo y Educación. 


\section{Revista de investigaciones UNAD \\ Volumen 10. Número 1. Junio 2011}

Congreso de Colombia. (1989). Código del menor. Bogotá.

Congreso de Colombia (2006).Código de infancia y adolescencia. Ley 1098. Bogotá.

De la Garza, M. T. (1995). Educación y Democracia. Madrid: Visor.

Delval, J. (1989). La representación infantil del mundo social. Buenos Aires: Alianza editorial.

Ginés, S. (1984). La cultura y el proceso de socialización. San Salvador: UCA Editores.

Guevara, M. (2000). Orientación educativa: contextualizar los problemas. Disponible en http://www .uco.es/-edlladip/revista/genios/ N1/ARTB1/Art34.htm. [2004, 20 de marzo].

Instituto Colombiano de Bienestar Familiar. (2004). Factores de riesgo sicosocial que caracterizan la problemática de los jóvenes infractores de la ley penal ubicados en el CROMI en la ciudad de Valledupar. Valledupar, Colombia.

Krauoskof, D. (1998). Dimensiones críticas en la participación social de juventudes. San J osé de Costa Rica: Fondo de Población de Naciones Unidas.

Martínez, M. (1989). El método de la historia de vida. Ciudad de México: Trillas.

Ministerio de Educación Nacional. (2004). Estándares Básicos de Competencias Ciudadanas. Revolución educativa: Colombia aprende. Bogotá.

Ortiz, A. (2005). Pedagogía Problémica. En Colombia 2009: Metodología del aprendizaje basado en problemas (pp.145-175). Bogotá: Cooperativa Editorial Magisterio.

Ortiz, E. Z. (2000). Así se Investiga. Pasos para hacer una investigación (2ª ed.). San Salvador: Clásicos Roxsil.

Ramírez, S., J. M. (1995). Las dimensiones de la Ciudadanía. Recuperado dehttp://148.202.18.157/sitios/publicacionesite/pperiod/espiral/espiralpdf/ Espiral2/89-112.pdf.

Restrepo, J . C. Estándares básicos en competencias ciudadanas: Una aproximación al problema de la formación ciudadana en Colombia: Papel político, 1, 137-175. Bogotá: Universidad J averiana.

Touraine, A. (1992). Critique de la modernité. París: Fayard.

Zubiría M. y Zubiría, J. (1987). Fundamentos de Pedagogía Conceptual. Bogotá: Plaza y J anés. 